\title{
Bin1 Antibodies for IBD Therapy: Is Permeability the Answer BAR None?
}

\author{
George Kolios ${ }^{1}$
}

Published online: 22 December 2015

(c) Springer Science+Business Media New York 2015

The inflammatory bowel diseases (IBD) ulcerative colitis (UC) and Crohn's disease (CD), of unknown etiology despite being extensively investigated over the last few decades, share important common pathogenic mechanisms. The interaction of genetic predisposing factors, exogenous and endogenous triggers, and modifying mediators induce inflammation and immune-mediated tissue injury. In UC, with inflammation mostly limited to the large bowel, acute and chronic inflammatory cells infiltrate the lamina propria with the formation of crypt microabscesses [1]. Recent diagnostic and therapeutic advances have been based on the increasing knowledge of the interplay between the cellular components and soluble mediators in the gut mucosa, combined with therapeutic strategies that target immunological mechanisms [2].

Drug therapy of IBD has traditionally been based on aminosalicylates, steroids, and immunomodulators which induce clinical remission without affecting the underlying disease. The first generation of immunomodulatory biologic therapy consisted of monoclonal antibodies directed against inflammatory mediators such as pro-inflammatory cytokines, against cell signaling inhibitors such as tyrosine kinase inhibitors, and biologic response modifiers such as immunomodulatory cytokines. The first biologic treatment of IBD, which has revolutionized treatment, targeted tumor necrosis factor- $\alpha$ (TNF- $\alpha$ ). The next generation of biologics, also targeting TNF- $\alpha$, was also effective in the treatment of refractory IBD, inducing longer remission and

George Kolios

gkolios@med.duth.gr

1 Laboratory of Pharmacology, Faculty of Medicine, Democritus University of Thrace, Dragana, 68100 Alexandroupolis, Greece mucosal healing, but is limited by multiple adverse effects, limiting its utility [2]. The most recent therapies target the integrity and functionality of the mucosa and its interaction with the luminal microflora, tailored to patients in different therapeutic groups based on pathogenesis rather than on clinical phenotype. Thus, in the "new age" of IBD therapy, hypothetical subgroups such as IBD1, IBD2, IBD3, and IBD4 would be treated rather than UC and CD [3].

IBD is thought to occur following a triggering event in a genetically predisposed host in a susceptible local environment. Along with immune dysregulation, impaired intestinal barrier function enhances inflammation or exacerbates experimental colitis, possibly mediated by the cytokines TNF- $\alpha$ and interleukin (IL)-13 [4], which suggests that therapy using systemic immunomodulatory agents combined with regulators of intestinal barrier function holds promise for enhanced efficacy.

In this issue of Digestive Diseases and Sciences, Thomas et al. [5] report that a monoclonal antibody targeting the bridging integrator 1 (Bin1) protein reduced colitis morbidity in a mouse model of experimental colitis, which they attributed in part to strengthening intestinal barrier function, based in part on a prior publication in which Bin1 mosaic knockout mice were resistant to chemically induced colitis [6]. Bin1 is a prototypic member of the Bin1/Amphihysin/Rvs (BAR) adapter gene family, a universally expressed nucleocytoplasmic protein consisting of numerous splice variants with differing tissue distribution, subcellular localization, and protein interactions that influence diverse cellular functions such as DNA repair, cell cycle progression, apoptosis, and cytoskeleton regulation. Bin1 was identified as a binding partner of the transcription factor c-Myc, an oncoprotein whose overexpression is associated with poor cancer prognosis [7]. Bin1, although implicated in the pathogenesis of serious pathology such as 
ventricular cardiomyopathy, arrhythmia preceding heart failure, and late-onset Alzheimer's disease, is most importantly linked with oncogenesis as a tumor suppressor [8].

Thomas et al. developed a monoclonal antibody that targets the C-terminal of Bin1 (Bin1 mAb) that significantly attenuated dextran sodium sulfate (DSS)-induced colitis in mice, reducing mucosal infiltration by inflammatory cells, preventing epithelial surface damage, and maintaining lymphoid follicle integrity. In vitro studies conducted in the intestine-derived adenocarcinoma $\mathrm{Caco} 2$ cell line demonstrated that the presence of Bin $1 \mathrm{mAb}$ protected tight junctional (TJ) structures and improved barrier function after treatment with the pro-inflammatory compound phorbol ester 12-O-tetradecanoylphorbol-13acetate, or the cytokines TNF- $\alpha$ and interferon (IFN)- $\gamma$. Nevertheless, since Bin1 suppresses the development and progression of colon adenocarcinoma due to its aforementioned tumor suppressor activity [9], its therapeutic administration could potentially augment the increased cancer risk observed in UC. Reduced expression of Bin1 in some tissues increases the activity of the immunomodulatory enzyme indoleamine 2,3 dioxygenase 1 (IDO1) that modulates inflammatory responses in tissue and supports carcinogenesis. This mechanism could further reduce the tumor suppressor activity of Bin1 [10]. The in vitro results of this study indicated that the effect of Bin1 mAb administration in cultured colonic epithelial cells did not increase the expression of IDO1. Given this differential effect of Bin $1 \mathrm{mAb}$ in colonic epithelial cells compared to other tissues, the authors suggested that its potential therapeutic use in ulcerative colitis might be accompanied by a reduction in the risk of colorectal cancer. Nevertheless, more preclinical data are needed in order to clarify the safety profile of Bin $1 \mathrm{mAb}$, as a potential therapeutic agent with the caveat that preclinical results may not translate fully to clinical studies [11].

The findings presented in the study by Thomas et al. provide new insights into the treatment of colitis in which strengthening of barrier function could ameliorate intestinal inflammation and prevent chronic relapses and perhaps cancer development. More studies targeting barrier dysfunction could clarify whether these results are just another experimental observation or signify a new era in IBD management.

\section{References}

1. Zhang YZ, Li YY. Inflammatory bowel disease: pathogenesis. World J Gastroenterol. 2014;20:91-99.

2. Cohen LB, Nanau RM, Delzor F, Neuman MG. Biologic therapies in inflammatory bowel disease. Transl Res. 2014;163: 533-556.

3. Bernstein CN. Treatment of IBD: where we are and where we are going. Am J Gastroenterol. 2015;110:114-126.

4. Mankertz J, Schulzke JD. Altered permeability in inflammatory bowel disease: pathophysiology and clinical implications. Curr Opin Gastroenterol. 2007;23:379-383.

5. Thomas S, Mercado JM, DuHadaway J, DiGuilio K, Mullin JM, Prendergast GC. Novel colitis immunotherapy targets Bin1 and improves colon cell barrier function. Dig Dis Sci. (Epub ahead of print). doi:10.1007/s10620-015-3804-8.

6. Chang MY, Boulden J, Valenzano MC, et al. Bin1 attenuation suppresses experimental colitis by enforcing intestinal barrier function. Dig Dis Sci. 2012;57:1813-1821.

7. Prokic I, Cowling BS, Laporte J. Amphiphysin 2 (BIN1) in physiology and diseases. J Mol Med (Berl). 2014;92:453-463.

8. Lundgaard GL, Daniels NE, Pyndiah S, et al. Identification of a novel effector domain of BIN1 for cancer suppression. $J$ Cell Biochem. 2011;112:2992-3001.

9. Chang MY, Boulden J, Katz JB, et al. Bin1 ablation increases susceptibility to cancer during aging, particularly lung cancer. Cancer Res. 2007;67:7605-7612.

10. Jia Y, Wang H, Wang Y, et al. Low expression of Bin1, along with high expression of IDO in tumor tissue and draining lymph nodes, are predictors of poor prognosis for esophageal squamous cell cancer patients. Int J Cancer. 2015;137:1095-1106.

11. Valatas V, Vakas M, Kolios G. The value of experimental models of colitis in predicting efficacy of biological therapies for inflammatory bowel diseases. Am J Physiol Gastrointest Liver Physiol. 2013;305:G763-G785. 